\title{
RESEARCH HIGHLIGHT Nesfatin-1 puts the brakes on reward-based feeding
}

\author{
Merridee J. Lefner ${ }^{1}$ and Matthew J. Wanat (D) \\ Neuropsychopharmacology (2020) 45:1591-1592; https://doi.org/10.1038/s41386-020-0695-y
}

The increasing prevalence of obesity is a significant public health concern, as it is associated with an increased risk of diabetes, heart disease, and stroke. Dysregulated feeding is a primary factor that contributes to obesity [1]. Food intake is controlled by both our caloric needs (homeostatic feeding) and our wants (reward-based feeding). Homeostatic feeding is primarily regulated by the hypothalamus, whereas reward-based feeding involves the mesolimbic dopamine system [2]. Previous research has focused on identifying the hunger and satiety signals within these systems [2]. This past decade has seen an emerging interest in the anorexigenic properties of the peptide nesfatin-1, which is a cleavage product of the protein nucleobindin-2 [3]. Endogenous nesfatin- 1 levels remain low during fasting and increase after feeding [3]. Nesfatin-1 has primarily been studied for its role within the homeostatic feeding system, as it is highly expressed in the hypothalamus [3]. However, nesfatin-1 is also expressed in reward-related brain regions [4], though it is unclear how nesfatin1 regulates reward-based feeding.

In their recent Neuropsychopharmacology article, Dore et al. [5] examined how nesfatin-1 influenced reward-based feeding using both operant tasks and preference assays. In the first set of experiments, they probed whether nesfatin-1 regulates the motivation to work for sucrose rewards. Mice were trained to lever press for the rewards under a progressive ratio reinforcement schedule, a common behavioral procedure for examining motivation. Intracerebroventricular (i.c.v.) injections of nesfatin-1 dose-dependently reduced the number of sucrose rewards earned under this reinforcement schedule. In contrast, i.c.v. injections of nesfatin-1 did not affect lever pressing or sucrose rewards earned when mice were trained using a low-effort fixed-ratio 1 reinforcement schedule. These data highlight that the central effect of nesfatin-1 on reward-based feeding is specific to high-effort tasks.

The mesolimbic dopamine system controls high-effort behaviors [6]. As such, Dore et al. examined whether the central effects of nesfatin-1 on motivation are due to its actions within the ventral tegmental area (VTA). To examine this possibility, mice received intra-VTA injections of nesfatin-1 before working for sucrose rewards under the progressive ratio reinforcement schedule. Just as was found with central injections, intra-VTA injections of nesfatin-1 reduced the number of sucrose rewards earned. These behavioral findings suggest that nesfatin-1 inhibits dopamine neurons. Indeed, the authors demonstrate that nesfatin- 1 is present in the VTA, and that VTA dopamine neurons are hyperpolarized by nesfatin-1.

In the second set of experiments, Dore et al. examined the effect of i.c.v. injections of nesfatin-1 on the preference between different reward options using a 2-bottle choice task. They demonstrated that nesfatin-1 injections reduced the preference for sucrose over water. This change in preference was due to a selective reduction in sucrose consumption, without affecting water consumption. Therefore, nesfatin- 1 does not globally impact fluid intake, rather it selectively attenuates the consumption of highly palatable fluids.

The final set of experiments examined the choice between sucrose and sucralose, a non-caloric solution that mimics the taste of sucrose. Mice reliably prefer sucrose over sucralose as there is a caloric benefit with consuming sucrose [7]. However, when sucralose consumption is paired with optogenetic stimulation of VTA dopamine neurons, ad libitum fed mice will instead prefer the sucralose + stimulation bottle over the sucrose bottle. Interestingly, if the mice are fasted prior to testing, the preference for sucralose + stimulation is lost, which likely reflects the higher caloric needs in a fasted state. Nesfatin-1 levels are lower after fasting and higher following feeding [3]. Therefore, high nesfatin-1 levels could mediate the preference for the sucralose + stimulation bottle in ad libitum fed mice. In support, Dore et al. demonstrate that i.c.v. injections of nesfatin- 1 in fasted mice induced a preference for the sucralose + stimulation option. These data highlight nesfatin- 1 can act centrally to bypass caloric needs to alter reward preference.

The findings by Dore et al. illustrate a role for nesfatin- 1 in the reward-based feeding system. While intra-VTA injections of nesfatin-1 diminished the motivation to work for sucrose in a progressive ratio task, it remains unclear what circumstances would induce the release of nesfatin- 1 endogenously within the VTA. Although nesfatin-1 enhanced the preference for sucralose paired with optogenetic simulation of dopamine neurons, these behavioral effects are likely not mediated at the level of the VTA and could involve additional reward-related regions such as the nucleus accumbens [8]. It is therefore imperative to further delineate the neural systems, as well as the specific neurons, that nesfatin- 1 acts on to regulate rewardbased feeding.

Maintaining proper feeding requires both the homeostatic and reward-based feeding systems. However, it should be noted that these systems are not necessarily independent of one another, as the hypothalamus sends projections to mesolimbic regions that mediate the pursuit of food and drug rewards [9]. This study, in combination with prior research, demonstrates that nesfatin-1 contributes to the cessation of feeding within both the homeostatic and reward-based feeding systems $[3,5]$. Moving forward, it will be important to elucidate the influence of nesfatin- 1 within the hypothalamic projections to the reward system. Importantly, there could be therapeutic relevance for nesfatin- 1 on both ends of the eating disorder spectrum. Increasing nesfatin-1 signaling could prevent pathological overeating that leads to obesity, whereas inhibiting nesfatin-1 signaling could serve as a potential treatment for anorexia.

\footnotetext{
${ }^{1}$ Neurosciences Institute and Department of Biology, University of Texas at San Antonio, San Antonio, TX 78249, USA
}

Correspondence: Matthew J. Wanat (Matthew.wanat@utsa.edu)

Received: 24 April 2020 Accepted: 27 April 2020

Published online: 6 May 2020 
FUNDING AND DISCLOSURE

MJW is supported by NIH DA042362. The authors declare no competing interests.

\section{AUTHOR CONTRIBUTIONS}

MJL and MJW wrote the manuscript.

\section{REFERENCES}

1. Moore CF, Sabino V, Koob GF, Cottone P. Neuropsychopharmacology. 2017;42: $1375-89$.
2. Erlanson-Albertsson C. Basic Clin Pharm Toxicol. 2005;97:61-73.

3. Schalla MA, Stengel A. J Endocr Soc. 2018;2:1188-206.

4. Goebel-Stengel M, Wang L, Stengel A, Tache Y. Brain Res. 2011;1396:20-34.

5. Dore R, Krotenko R, Reising JP, Murru, L, Sundaram SM, Spiezio AD, et al. Neuropsychopharmacology. 2020. https://doi.org/10.1038/s41386-020-0682-3.

6. Salamone JD, Correa M, Mingote S, Weber SM. J Pharm Exp Ther. 2003;305:1-8.

7. de Araujo IE, Oliveira-Maia AJ, Sotnikova TD, Gainetdinov RR, Caron MG, Nicolelis $M A$, et al. Neuron. 2008;57:930-41.

8. Chen X, Shu X, Cong, ZK, Jiang ZY, Jiang, H. Neuropeptides. 2015;53:45-50.

9. Watabe-Uchida M, Zhu L, Ogawa SK, Vamanrao A, Uchida N. Neuron. 2012;74:858-73. 